\title{
Innovationspreis 2015
}

\section{Ring frei für die fünfte Runde}

\author{
Innovationen haben immer Konjunktur. Das gilt auch und besonders \\ für niedergelassene Ärzte, die angesichts steigender Patientenzahlen \\ und strikter Budgetgrenzen viel Kreativität benötigen, um den Spaß \\ an der Arbeit zu behalten und eine wirtschaftliche Praxisführung zu \\ gewährleisten. Für den Wettbewerb „Die innovative Arztpraxis" sind \\ daher auch 2015 wieder Ärzte mit guten Ideen für die Praxis gefragt.
}

$\mathrm{D}$ er Wettbewerb „Die innovative Arztpraxis" geht 2015 nun schon in die fünfte Runde. Initiiert von der Fachverlagsgruppe Springer Medizin und dem Biopharmaunternehmen UCB ist er für Arztpraxen gedacht, die mit pfiffigen Ideen, veränderter Praxisorganisation, effizienter Umsetzung von Vorgaben aus Politik und Selbstverwaltung sowie mit innovativen medizinischen Angeboten aus der Masse herausragen. Kreativität ist dabei in vielen $\mathrm{Ar}$ beitsbereichen der Praxis denkbar:

- Bei drohender Unterversorgung auf dem Land wächst der Versorgungsdruck. Mehr Patienten müssen von weniger Ärzten versorgt werden. Hier sind Ideen in der Steuerung der Patienten ebenso gefragt wie der Mut zur Delegation von Aufgaben an MFA.

-Um sich im Wettbewerb der Praxen weiter gut zu behaupten, könnten neue Konzepte für die Personalführung von Nutzen sein oder auch der Einsatz moderner Technik, die Patienten den Kontakt zur Praxis erleichtern oder Ärzten bei der Dokumentation helfen. - Mit Aktivitäten in sozialen Medien oder mit kleinen Programmen für das Handy von Patienten können die $\mathrm{Pa}$ tientenbindung und die Adhärenz der Patienten gestärkt werden.

_Oft sind es die kleinen Ideen, die Ärzten in der Praxis das Leben leichter machen können, kleine Handreichungen oder Umstrukturierungen in der Praxisorganisation - nicht nur die großen Erfindungen, neue Geräte oder große Computerprogramme.
„Eine optimale Versorgung für die Patienten, die aber auch für Ärzte machbar bleibt und wirtschaftlich ist: Praxen, die diesem Ziel durch gute Ideen einen Schritt näher kommen, haben beste Chancen, den Innovationspreis zu gewinnen", sagt Peter Mitterhofer, Geschäftsführer von UCB in Deutschland.

\section{Unterschiedliche Ansätze für einen besseren Praxiserfolg}

Dass niedergelassene Vertragsärzte ein hohes Kreativitäts- und Innovationspotenzial haben, wenn es um neue Ansätze der Patientenversorgung geht, zeigt ein Blick zurück auf die ersten Runden des Wettbewerbs. Die Sieger in den vergangenen Jahren:

_Die hausärztliche Internistin Irmgard Landgraf aus Berlin wurde für die effiziente telemedizinische Versorgung eines Pflegeheims im Jahr 2011 ausgezeichnet. Das Konzept hat inzwischen Schule gemacht, Frau Landgraf berät mittlerweile auch Kollegen bei der Umsetzung eines solchen Konzepts.

_ 2012 gewannen die Ärzte Dr. Herbert Baake, Dr. Jan Purr, Dr. Robert Tanu und Dr. Nicole Baake-Möller aus dem Gesundheitszentrum Gelstertal den 1. Preis - mit ihrer Strategie zur optimierten Versorgung von Patienten im ländlichen Raum.

_2013 ging der Preis an den Neurologen Dr. Falk von Zitzewitz aus Ludwigsburg. Er hat ein elektronisches Patiententagebuch entwickelt, mit dem Patienten ihre Befindlichkeit minutenge-

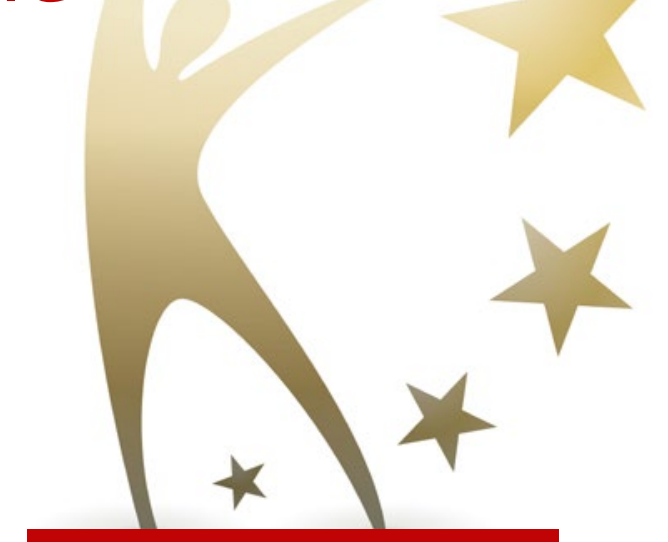

"Die innovative Arztpraxis 2015"

Mitmachen lohnt sich!

Allen Wettbewerbsteilnehmern winken wertvolle Preise. Der Sieger erhält das Befragungstool HCC Analytics, finanziert und durchgeführt von der Unternehmensberatung HCC Better Care in Köln. Die Preisträger werden wie in den Vorjahren von einer unabhängigen Jury und von Internet-Nutzern auf "Ärzte Zeitung online" bestimmt.

Bewerben können sich Praxisteams online unter:

www.aerztezeitung.de/ innovationspreis

Bewerbungsschluss ist der 30. November 2015. Die Bekanntgabe der Gewinner findet Anfang 2016 statt.

nau dokumentieren können. Dem behandelnden Arzt erschließen sich dadurch wertvolle Zusatzinformationen. _Letztes Jahr schließlich haben die Dermatologinnen Stefanie Baum und ihre Praxispartnerin Dr. Sonja Ehlen aus Kassel den 1. Preis gewonnen. In ihrer Praxis füllen Patienten bereits im Wartezimmer Aufklärungsformulare und Erstanamnesebögen aus, die Dokumentation erfolgt direkt in der Praxis-EDV. Das spart viel Zeit beim Arzt-Patienten-Kontakt.

Lassen Sie sich durch die Ideen der ersten Preisträger inspirieren und machen Sie mit!

Hauke Gerlof 